\title{
Glucocorticoid receptor antagonism decreases alcohol seeking in alcohol-dependent individuals
}

\author{
Leandro F. Vendruscolo, ${ }^{1}$ David Estey, ${ }^{1}$ Vivian Goodell, ${ }^{1}$ Lauren G. Macshane, ${ }^{1}$ Marian L. Logrip, ${ }^{1}$ Joel E. Schlosburg, ${ }^{1}$ \\ M. Adrienne McGinn, ${ }^{2}$ Eva R. Zamora-Martinez, ${ }^{1}$ Joseph K. Belanoff, ${ }^{3}$ Hazel J. Hunt, ${ }^{3}$ Pietro P. Sanna, ${ }^{1}$ Olivier George, ${ }^{1}$ \\ George F. Koob, ${ }^{1}$ Scott Edwards, ${ }^{2}$ and Barbara J. Mason ${ }^{1}$
}

${ }^{1} T$ The Scripps Research Institute, La Jolla, California, USA. ²Department of Physiology and Alcohol and Drug Abuse Center of Excellence, LSU Health Sciences Center, New Orleans, Louisiana, USA. ${ }^{3}$ Corcept Therapeutics, Menlo Park, California, USA.

\begin{abstract}
Alcoholism, or alcohol use disorder, is a major public health concern that is a considerable risk factor for morbidity and disability; therefore, effective treatments are urgently needed. Here, we demonstrated that the glucocorticoid receptor (CR) antagonist mifepristone reduces alcohol intake in alcohol-dependent rats but not in nondependent animals. Both systemic delivery and direct administration into the central nucleus of the amygdala, a critical stress-related brain region, were sufficient to reduce alcohol consumption in dependent animals. We also tested the use of mifepristone in $\mathbf{5 6}$ alcoholdependent human subjects as part of a double-blind clinical and laboratory-based study. Relative to placebo, individuals who received mifepristone ( $600 \mathrm{mg}$ daily taken orally for 1 week) exhibited a substantial reduction in alcohol-cued craving in the laboratory, and naturalistic measures revealed reduced alcohol consumption during the 1-week treatment phase and 1-week post-treatment phase in mifepristone-treated individuals. Mifepristone was well tolerated and improved liverfunction markers. Together, these results support further exploration of GR antagonism via mifepristone as a therapeutic strategy for alcoholism.
\end{abstract}

\section{Introduction}

Alcohol misuse accounts for approximately $6 \%$ of deaths worldwide and is one of the largest risk factors for morbidity and disability (1). Alcoholism, or alcohol use disorder, is a complex psychiatric condition characterized by craving, compulsive seeking, and excessive drinking of alcohol, as well as the emergence of negative emotional states during withdrawal (2-4). Allostatic changes in brain reward and stress systems are thought to mediate many of these symptoms. We previously reported that dysregulation of the hypothalamic-pituitary-adrenal (HPA) axis, the principal neuroendocrine stress system, and consequent alterations in brain glucocorticoid receptor (GR) expression accompany compulsive-like alcohol intake in rats $(5,6)$. Despite these strong preclinical findings and evidence for dysregulation of the HPA axis in alcoholism $(7,8)$, a role for GR signaling in humans who suffer from alcohol dependence has yet to be determined. Here, employing an integrative conceptual approach to medication development for alcohol use disorder (9), we examined the hypothesis that altered GR signaling mediates compulsive alcohol intake in both rats and humans.

\section{Results and Discussion}

We first tested the hypothesis that GRs were activated during alcohol dependence by employing an animal model that produces somatic and motivational signs of alcoholism strongly resembling the human condition $(10,11)$. The levels of phosphorylated

Conflict of interest: The authors have declared that no conflict of interest exists. Submitted: November 3, 2014; Accepted: May 21, 2015.

Reference information: J Clin Invest. 2015;125(8):3193-3197. doi:10.1172/JCI79828. and total GR were measured in the central amygdala (CeA) and basolateral amygdala (BLA), two brain regions critically implicated in stress, relapse, and the transition to drug addiction (12). Rats were made dependent by exposure to alcohol vapor, with peak blood alcohol levels averaging $237.0 \mathrm{mg} \% \pm 11.3 \mathrm{mg} \%$ in the 12th and final week of exposure. Air-exposed rats served as nondependent controls. Brain tissue collection occurred during acute withdrawal (6-8 hours after removal from alcohol vapor) when brain and blood alcohol levels are negligible (13) and withdrawal symptoms - including anxiety and brain reward deficits - are manifest $(10,11,14)$. Previous work has established that, under these conditions, nondependent rats will work to obtain and ingest $10 \% \mathrm{wt} / \mathrm{vol}$ alcohol, and dependent rats will escalate their intake sufficient to block withdrawal and show compulsivelike responding for alcohol $(6,10,15)$. We found that the levels of GR phosphorylation at $\mathrm{Ser}^{232}$, a marker of GR nuclear localization and transactivation, were increased in the CeA $\left(t_{10}=2.574\right.$, $P=0.0277)$ but not the adjacent BLA $\left(t_{10}=0.486, P=0.8087\right)$ in alcohol-dependent rats compared with nondependent rats (Figure 1A). Total GR levels were unchanged, suggesting an increase in physiological GR function, rather than total receptor number in the $\mathrm{CeA}$ in the transition to alcohol dependence.

To determine the functional significance of the activation of GRs in alcohol dependence, we tested a GR antagonist, mifepristone, on compulsive-like alcohol intake. Rats were trained to lever press for access to alcohol (10\%, wt/vol) or water in an operant task, and half were subsequently made dependent by exposure to alcohol vapor. Dependent and nondependent rats ( $n=11$ /group) were i.p. injected with mifepristone (0, 30, and $60 \mathrm{mg} / \mathrm{kg}$ ) 90 minutes prior to alcohol self-administration ses- 

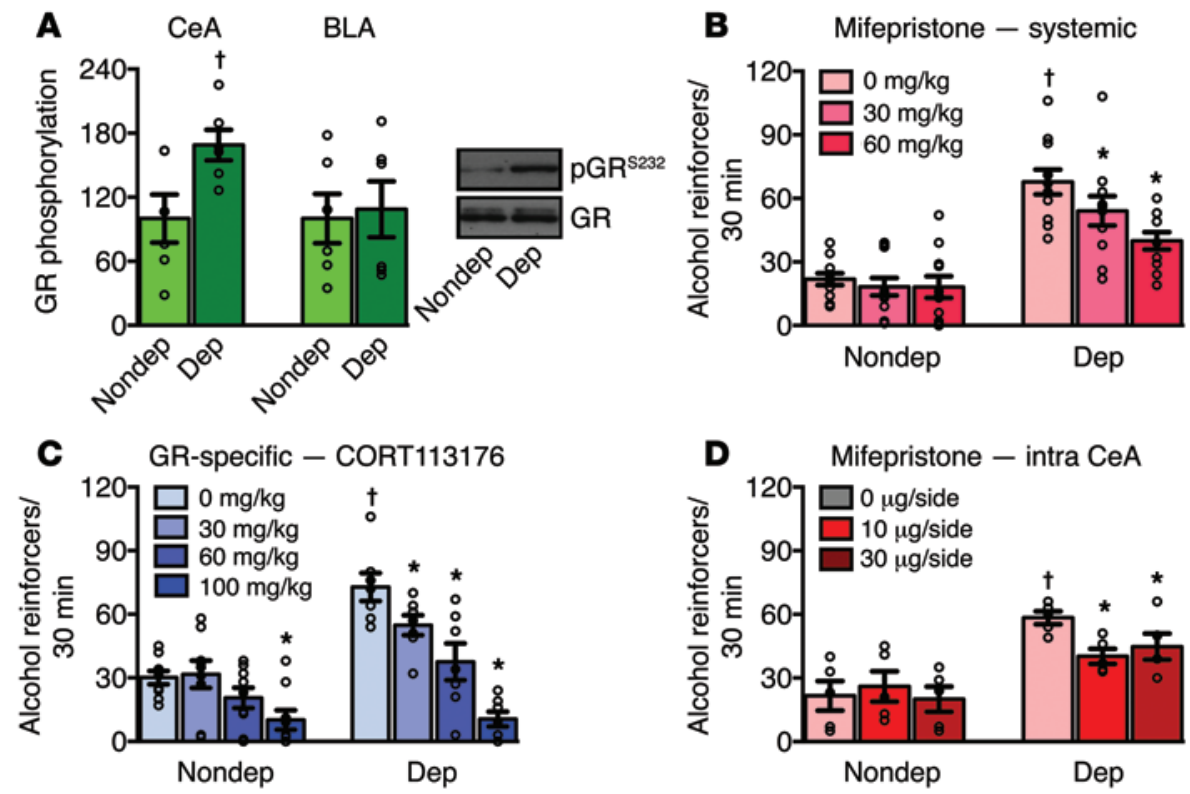

Figure 1. Increased GR function mediates compulsive-like alcohol self-administration in alcohol-dependent rats. (A) The phosphorylation of GR at Ser ${ }^{5232}$ in the CeA (2-tailed Student's $t$ test, ${ }^{\dagger} P<0.05$ versus nondep), but not BLA, was increased in dependent (dep) rats compared with nondependent (nondep) rats ( $n=6 /$ group). No group differences in total GR levels were observed. Representative Western blots for phosphorylated and total GRs in the CeA are indicated. (B) The blockade of GRs with mifepristone injected systemically ( $n=11$ /group) dose-dependently reduced alcohol intake, specifically in dep rats. (C) The GR-specific antagonist CORT113176 decreased alcohol self-administration ( $n=7$ for dep and $n=9$ for nondep), with increased effectiveness in dep rats. (D) The blockade of GRs with mifepristone injected directly into the CeA ( $n=5$ /group) reduced alcohol intake, specifically in dep rats. (B-D) 2-way repeated-measure ANOVA followed by Duncan's test $\left({ }^{*} P<0.05 \mathrm{vs} .0 \mathrm{mg} / \mathrm{kg},{ }^{+} P<0.0001\right.$ vs. nondep). The data represent the mean \pm SEM of the number of lever presses performed by the rats in 30-minute operant self-administration sessions. Drugs were injected 90 minutes prior to self-administration sessions. Every lever press (fixed-ratio 1 schedule of reinforcement) resulted in the delivery of $0.1 \mathrm{ml}$ of alcohol (10\%, wt/vol) or water. Dotplots display individual observations for each condition.

sions occurring during acute withdrawal. Dependent rats displayed escalated alcohol intake $(P=0.0001)$, i.e., significantly increased alcohol intake compared with nondependent rats. Systemic injection with mifepristone dose-dependently (dose $\times$ group interaction: $\left.F_{2,40}=4.675, P=0.0150\right)$ reduced alcohol intake in dependent rats only $(P=0.0199,0$ vs. $30 \mathrm{mg} / \mathrm{kg}$; $P=0.0001,0$ vs. $60 \mathrm{mg} / \mathrm{kg}$; Figure 1B). These findings provide evidence that acute mifepristone administration, in addition to blocking the development of compulsive-like alcohol drinking (6), significantly decreases alcohol self-administration when dependence has already been established. Our results corroborate and extend previous studies indicating that mifepristone decreases both alcohol drinking under limited-access conditions (16) and the severity of alcohol withdrawal (17).

Because mifepristone potently inhibits both GR and progesterone receptor function, we tested the effect of a selective GR antagonist on alcohol drinking. CORT113176 antagonizes GRs comparably to mifepristone but has no affinity for progesterone receptors (Supplemental Table 1; supplemental material available online with this article; doi:10.1172/JCI79828DS1). CORT113176 dose-dependently ( $n=7$ for dependent; $n=9$ for nondependent; dose $\times$ group interaction: $\left.F_{3,42}=5.168, P=0.0039\right)$ decreased alcohol intake (Figure 1C) at doses of $30 \mathrm{mg} / \mathrm{kg}(P=0.0233)$ and 60 $\mathrm{mg} / \mathrm{kg}(P=0.0001)$ compared with vehicle $(0 \mathrm{mg} / \mathrm{kg})$ in depen- dent rats only, while it reduced drinking at $100 \mathrm{mg} / \mathrm{kg}$ in both dependent $(P=$ $0.0001)$ and nondependent $(P=0.0194)$ animals. Vehicle-treated dependent rats displayed escalated alcohol intake compared with vehicle-treated nondependent rats $(P=0.0001)$. Additional control experiments indicated that mifepristone and CORT113176 had no effect on water or saccharin intake (Supplemental Figure 1). These results provide clear-cut evidence for a specific role of GRs in the escalation of alcohol drinking that does not generalize to nondrug rewards. This selectivity would predict robust and specific applicability of mifepristone to treat human alcoholism, with a critical new feature not encountered in existing medications, such as naltrexone (18), that act on reward mechanisms.

To confirm a key role of the CeA in the glucocorticoid antagonist effect, dependent and nondependent rats were bilaterally injected with mifepristone $(0,10$, and $30 \mu \mathrm{g} / \mathrm{side})$ directly into the CeA 90 minutes prior to alcohol selfadministration. Vehicle-treated dependent rats displayed escalated alcohol intake compared with vehicle-treated nondependent rats $(P=0.0007)$. Mifepristone $(n=5 /$ group; dose $\times$ group interaction: $\left.F_{2,16}=4.044, P=0.0379\right)$ at $10 \mu \mathrm{g} / \mathrm{side}$ $(P=0.0068)$ and $30 \mu \mathrm{g} / \mathrm{side}(P=0.0280)$, significantly and specifically reduced alcohol intake in dependent rats, when compared with vehicle $(0 \mu \mathrm{g} / \mathrm{side})$ controls (Figure 1D). Our demonstration of increased GR phosphorylation during dependence and reduction of compulsive-like alcohol drinking by intra-CeA mifepristone injection, combined with previous findings (19), indicate that increased GR signaling in the CeA plays a functional role in alcohol drinking and seeking.

For clinical validation of our findings, we employed a human laboratory model of risk factors for relapse in abstinence in nontreatment-seeking, paid, alcohol-dependent volunteers (20). The subjects (Supplemental Figure 2) were randomly assigned to double-blind dosing with oral mifepristone (600 $\mathrm{mg} /$ day, no titration) or placebo for 7 days. Alcohol abstinence was required during the last 3 days of the 7-day dosing period in order to test the effect of mifepristone when motivational signs of early abstinence are manifest. Alcohol cue reactivity manipulations, followed by craving ratings, were conducted in the laboratory at the conclusion of the 7-day dosing/3-day abstinent interval (see Supplemental Table 2). Craving in response to alcohol cues in abstinent alcoholics has been shown to be predictive of subsequent drinking relapse (21), and thus craving severity in response to alcohol cue exposure in the laboratory comprised the primary outcome for this proof-ofconcept study. Subjects returned 1 week after drug discontinuation to assess any persisting drug effects on safety indices and on nat- 

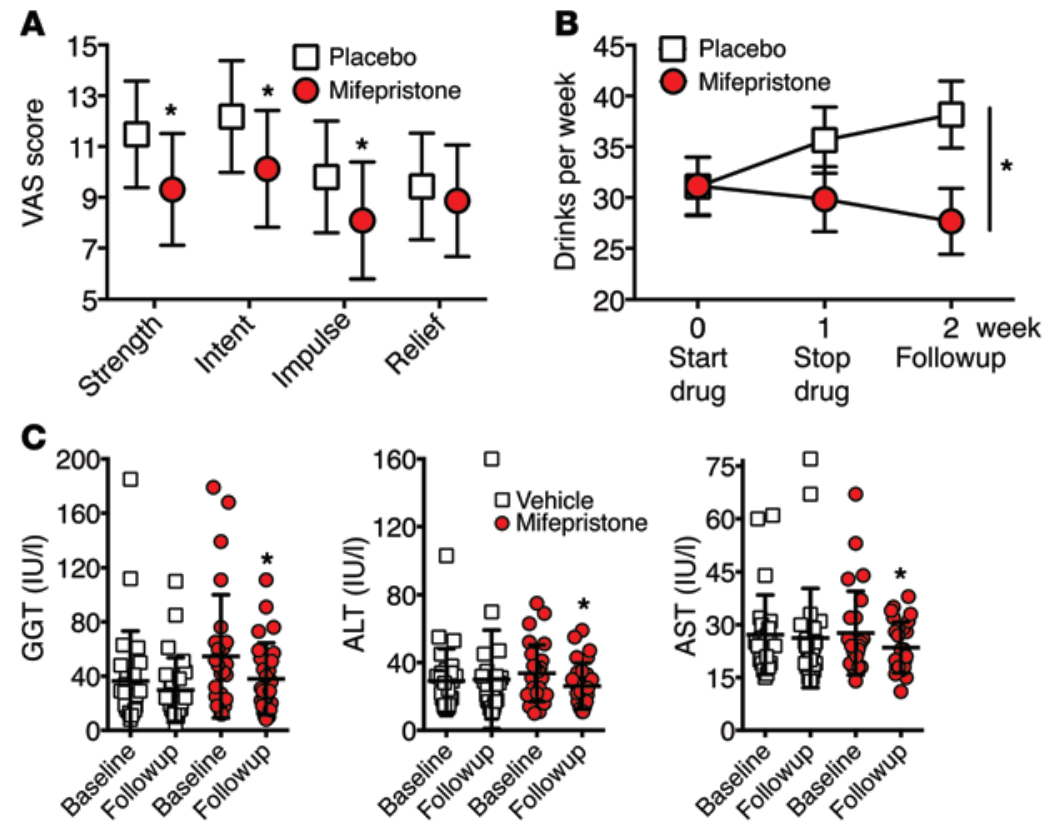

Figure 2. Mifepristone reduces alcohol-cued craving and drinking in alcoholics while improving liver function.

(A) Visual Analog Scale (VAS) scores of craving severity in response to in vivo alcohol cues. Higher scores indicate greater craving severity. Data represent the estimated marginal mean $\pm \mathrm{SD}$. ${ }^{*} P<0.05$, mifepristone vs. placebo (linear mixed effects modeling). (B) Total number of alcoholic drinks consumed per week. Data represent the estimated marginal mean \pm SEM. ${ }^{*} P<0.05$, mifepristone vs. placebo (linear mixed effects modeling). (C) Liver enzymes: GGT, ALT, and AST. Data represent the mean $\pm S D$. Dotplots display individual observations for each condition. ${ }^{*} P<0.05$ within-group change from baseline (multivariate analysis of covariance). $n=26$ for placebo and $n=28$ for mifepristone. uralistic measures of drinking. Self-reported drinking data were collected on an exploratory basis, as the 3-day abstinent interval constrained complete evaluation of this variable, and drinking data were collected under less controlled conditions than craving in response to in vivo cue exposure. The subjects were 43 male and 13 female non-treatment-seeking volunteers, 21-65 years of age, who met the DSM-IV criteria for current alcohol dependence (see Supplemental Table 3 for baseline subject data). Mifepristone was associated with a significantly greater reduction in alcohol-cued craving in the laboratory relative to placebo after 1 week of treatment (sum score of the four VAS craving items: $-6.43, t=-3.01$, $P=0.003$; Figure 2A). Mifepristone was also associated with a significantly greater reduction in the number of drinks per week during treatment and post-treatment followup compared with placebo $(-10.58, t=-1.99, P=0.05$; Figure $2 \mathrm{~B})$. The reduction in alcohol-cued craving in the laboratory significantly predicted a reduction in the number of drinks per drinking day at post-treatment followup $\left(\mathrm{R}^{2}=0.11, P=0.017\right.$ excluding 1 outlier, the full dataset $\mathrm{R}^{2}=0.15, P=0.005$; Supplemental Figure 4 ), thus lending support to the predictive validity of the human laboratory model.

Excessive alcohol drinking is typically associated with elevation in liver-function markers, including aspartate aminotransferase (AST), alanine aminotransferase (ALT), and $\gamma$-glutamyltransferase (GGT). Biochemical tests of liver-function markers indicated significant reductions of GGT, ALT, and AST from baseline to followup in the mifepristone-treated group $(F=5.46, P=$ $0.005)$ but not in the placebo group $(F=1.60, P=0.218$; Figure $2 \mathrm{C})$. The reductions in these physiological markers in the treated group corroborate the self-reported decreases in drinking and provide evidence of the hepatic safety of mifepristone in alcoholics. Whether mifepristone directly reduces liver-function markers remains to be determined.

No serious or unexpected adverse events were observed, and the groups did not differ in the type or severity of adverse events during treatment (Supplemental Table 4). No effects on measures of mood were found. The effects of mifepristone did not resemble alcohol or any major classes of abused drugs (Supplemental Figure 3), thus excluding the possibility that drug substitution could explain the effects of mifepristone on alcohol craving and drinking, and mitigating against its abuse potential.

The motivation to drink alcohol is initially driven by its pleasurable/euphorigenic effects via positive reinforcement, and glucocorticoids can facilitate these effects (22). However, during dependence, both the HPA axis and central brain reward systems are dysregulated, and extrahypothalamic stress systems become sensitized, possibly mediated by the GR signaling changes reported herein that putatively drive drinking via negative reinforcement (i.e., stress relief). The molecular mechanisms responsible for mifepristone's ability to reduce compulsive alcohol drinking may involve altered gene expression of stress-related neuropeptide systems (e.g., corticotropin-releasing factor [CRF] and/or vasopressin) $(23,24)$. For example, acutely, high blood glucocorticoid levels are paralleled by enhanced GR-mediated CRF release in the CeA (25). Chronically, high glucocorticoid levels increase CRF expression in the CeA, whereas glucocorticoids inhibit CRF and vasopressin expression in the paraventricular nucleus of the hypothalamus (PVN) (26).

The rationale for the translational strategy between animal and human experiments in this study is focused on the dual role of glucocorticoids in suppressing HPA axis responses at the same time as sensitizing $\mathrm{CeA}$ responses, both of which are adaptive when an organism is chronically stressed. One could speculate that, under chronic stress, from a survival perspective, the blunted HPA response will prevent Cushing's disease-like symptoms, but the sensitized amygdala retains alertness for danger. A similar chronic stress-like profile also characterizes alcoholism. While alcohol can acutely activate the HPA axis via elevated hypothalamic production of CRF, more importantly, glucocorticoids are chronically released during the transition to alcohol dependence and then feedback to shut off the HPA axis and sensitize CRF in 
the amygdala. We believe that this CRF overactivity in the amygdala drives negative reinforcement to cause excessive drinking during dependence, as we know that CRF antagonists injected into the amygdala can block dependence-induced drinking and dysregulated cellular plasticity within the amygdala $(23,27)$. Thus, although activation of the HPA axis may characterize initial drug use within the binge/intoxication stage of addiction, such engagement can also lead to subsequent activation of extrahypothalamic brain stress systems that characterize the withdrawal/negativeaffect stage and protracted abstinence in the preoccupation/anticipation stage (2). Our hypothesis is that mifepristone can exhibit its potential therapeutic effects by restabilizing negative feedback along the HPA axis and blocking the sensitization of extrahypothalamic CRF in the amygdala (28). Future investigations at the preclinical and clinical levels should determine the precise stress and reward circuitry mechanisms of GR activity, including the role of epigenetic, genomic, and nongenomic GR signaling (29).

The present studies indicate that attenuation of GR function by mifepristone reduces compulsive-like alcohol intake in alcoholdependent rats and reduces both excessive drinking and alcohol craving in recently abstinent alcoholics - in addition to improving liver-function markers in subjects with a history of heavy drinking - without any major adverse effects. We suggest that brief (1-week) treatment with mifepristone immediately following acute withdrawal, in conjunction with a course of psychosocial treatment, may offer a novel therapeutic approach for alcohol dependence that optimizes healthcare resources. An adequately powered clinical trial of mifepristone to replicate and extend these findings to treatment seekers with alcohol dependence is indicated.

\section{Methods}

Further information can be found in Supplemental Methods, Supplemental Figures 1-4, and Supplemental Tables 1-4.

Statistics. Preclinical data were analyzed using 2-tailed Student's $t$ test or using repeated-measures ANOVA followed by Duncan's multiple comparison test. The clinical data were analyzed using 2-tailed Student's $t$ test, $\chi^{2}$ test, repeated-measures ANOVA, Pearson's product moment correlation, linear regression, multivariate analysis of covariance, and linear mixed effects modeling. The accepted level of significance for all of the tests was $P<0.05$.
Study approval. The preclinical procedures adhered to the National Institutes of Health Guide for the Care and Use of Laboratory Animals and were approved by the Institutional Animal Care and Use Committee of The Scripps Research Institute. Our single-site outpatient study was conducted in accordance with the Declaration of Helsinki. The Scripps Institutional Review Board approved the protocol, which was conducted under an investigator-initiated investigational new drug (IND) granted by the federal Food and Drug Administration. A Certificate of Confidentiality was obtained from the National Institute on Alcohol Abuse and Alcoholism to protect the confidentiality of participants. Written informed consent was obtained from all participants.

Trial registration: NCT01548417 (clinicaltrials.gov).

\section{Acknowledgments}

Research was financially supported by National Institute on Alcohol Abuse and Alcoholism (NIAAA/NIH) grants AA012602 and AA023152 (to B.J. Mason), AA020608, AA022977, and AA006420 (to O. George), and AA020839 (to S. Edwards), the Pearson Center for Alcoholism and Addiction Research, and LSUHSC School of Medicine Start-Up Funds (to S. Edwards). The authors thank Jordan Malavar and Muhammad Farooq for technical assistance with behavioral and biochemical measures, Michael Arends for editorial assistance, and Farhad Shadan, Mark Kyle, and Adnan Begovic for providing medical coverage for the participants in the clinical study.

Address correspondence to: Leandro F. Vendruscolo, NIH/ NIDA - IRP/INRB, 251 Bayview Blvd., BRC Room 08A727, Baltimore, Maryland 21224, USA. Phone: 443.740.2869; E-mail: leandro.vendruscolo@nih.gov. Or to: Barbara J. Mason, The Scripps Research Institute, 10550 N. Torrey Pines Road, TPC-5, La Jolla, California 92037, USA. Phone: 858.784.7328; E-mail: mason@scripps.edu.

Leandro F. Vendruscolo's present address is: National Institute on Drug Abuse, NIH, Baltimore, Maryland, USA.

George F. Koob's present address is: National Institute on Alcohol Abuse and Alcoholism, NIH, Washington, DC, USA.
1. WHO. Global Status Report On Alcohol And Health. Geneva, Switzerland: World Health Organization; 2014

2. Koob GF. Theoretical frameworks and mechanistic aspects of alcohol addiction: alcohol addiction as a reward deficit disorder. Curr Top Behav Neurosci. 2013;13:3-30.

3. Egli M, Koob GF, Edwards S. Alcohol dependence as a chronic pain disorder. Neurosci Biobehav Rev. 2012;36(10):2179-2192.

4. Mason BJ, Higley AE. A translational approach to novel medication development for protracted abstinence. Curr Top Behav Neurosci. 2013;13:647-670.

5. Richardson HN, Lee SY, O'Dell LE, Koob GF, Rivier CL. Alcohol self-administration acutely stimulates the hypothalamic-pituitary-adrenal axis, but alcohol dependence leads to a damp- ened neuroendocrine state. Eur J Neurosci. 2008;28(8):1641-1653.

6. Vendruscolo LF, et al. Corticosteroid-dependent plasticity mediates compulsive alcohol drinking in rats. J Neurosci. 2012;32(22):7563-7571.

7. Wand G, Dobs AS. Alterations in the hypothalamicpituitary-adrenal axis in actively drinking alcoholics. JClin Endocrinol Metab. 1991;72(6):1290-1295.

8. Uhart M, Wand GS. Stress, alcohol and drug interaction: an update of human research. Addict Biol. 2009;14(1): 43-64.

9. Koob GF, Kenneth Lloyd G, Mason BJ. Development of pharmacotherapies for drug addiction: a Rosetta stone approach. Nat Rev Drug Discov. 2009;8(6):500-515.

10. Vendruscolo LF, Roberts AJ. Operant alcohol self-administration in dependent rats: focus on the vapor model. Alcohol. 2014;48(3):277-286.
11. Meinhardt MW, Sommer WH. Postdependent state in rats as a model for medication development in alcoholism. Addict Biol. 2015;20(1):1-21.

12. Edwards S, Koob GF. Neurobiology of dysregulated motivational systems in drug addiction. Future Neurol. 2010;5(3):393-401.

13. Gilpin NW, Smith AD, Cole M, Weiss F, Koob GF, Richardson HN. Operant behavior and alcohol levels in blood and brain of alcohol-dependent rats. Alcohol Clin Exp Res. 2009;33(12):2113-2123.

14. Schulteis G, Markou A, Cole M, Koob GF. Decreased brain reward produced by ethanol withdrawal. Proc Natl Acad Sci US A. 1995;92(13):5880-5884.

15. Roberts AJ, Cole M, Koob GF. Intra-amygdala muscimol decreases operant ethanol self-administration in dependent rats. Alcohol Clin Exp Res. 1996;20(7):1289-1298. 
16. Koenig HN, Olive MF. The glucocorticoid receptor antagonist mifepristone reduces ethanol intake in rats under limited access conditions. Psychoneuroendocrinology. 2004;29(8):999-1003.

17. Sharrett-Field L, Butler TR, Berry JN, Reynolds AR, Prendergast MA. Mifepristone pretreatment reduces ethanol withdrawal severity in vivo. Alcohol Clin Exp Res. 2013;37(8):1417-1423.

18. Swift R, Oslin DW, Alexander M, Forman R. Adherence monitoring in naltrexone pharmacotherapy trials: a systematic review. J Stud Alcohol Drugs. 2011;72(6):1012-1018.

19. Simms JA, Haass-Koffler CL, Bito-Onon J, Li R, Bartlett SE. Mifepristone in the central nucleus of the amygdala reduces yohimbine stress-induced reinstatement of ethanol-seeking. Neuropsychopharmacology. 2012;37(4):906-918.

20. Mason BJ, Light JM, Escher T, Drobes DJ. Effect of positive and negative affective stimuli and beverage cues on measures of craving in non treatment-seeking alcoholics. Psychopharmacol- ogy (Berl). 2008;200(1):141-150.

21. Cooney NL, Litt MD, Morse PA, Bauer LO, Gaupp

L. Alcohol cue reactivity, negative-mood reactivity, and relapse in treated alcoholic men. JAbnorm Psychol. 1997; 106(2):243-250.

22. Fahlke C, Hard E, Eriksson CJ, Engel JA, Hansen $\mathrm{S}$. Consequence of long-term exposure to corticosterone or dexamethasone on ethanol consumption in the adrenalectomized rat, and the effect of type I and type II corticosteroid receptor antagonists. Psychopharmacology (Berl). 1995;117(2):216-224.

23. Funk CK, O'Dell LE, Crawford EF, Koob GF. Corticotropin-releasing factor within the central nucleus of the amygdala mediates enhanced ethanol self-administration in withdrawn, ethanol-dependent rats. JNeurosci. 2006;26(44):11324-11332.

24. Edwards S, Guerrero M, Ghoneim OM, Roberts E, Koob GF. Evidence that vasopressin V1b receptors mediate the transition to excessive drinking in ethanol-dependent rats. Addict Biol. 2012;17(1):76-85.

25. Cook CJ. Glucocorticoid feedback increases the sensitivity of the limbic system to stress. Physiol Behav. 2002;75(4):455-464.

26. Sawchenko PE. Evidence for a local site of action for glucocorticoids in inhibiting CRF and vasopressin expression in the paraventricular nucleus. Brain Res. 1987;403(2):213-223.

27. Roberto M, Cruz MT, Gilpin NW, et al. Corticotropin releasing factor-induced amygdala $\gamma$-aminobutyric Acid release plays a key role in alcohol dependence. Biol Psychiatry. 2010;67(9):831-839.

28. Heilig M, Koob GF. A key role for corticotropin-releasing factor in alcohol dependence. Trends Neurosci. 2007;30(8):399-406.

29. Tasker JG, Herman JP. Mechanisms of rapid glucocorticoid feedback inhibition of the hypothalamic-pituitary-adrenal axis. Stress. 2011;14(4):398-406. 